\title{
FRICTIONAL FORCE MODELING OF A MACHINE TOOL FEED DRIVE TELESCOPIC COVER
}

J. Ferkl ${ }^{1 *}$, L. Novotny ${ }^{1}$, S. Fiala ${ }^{1}$, P. Kolar ${ }^{1}$, M. Rytir ${ }^{1}$

${ }^{1}$ Czech Technical University in Prague, Faculty of Mechanical Engineering, Department of Production Machines and Equipment (RCMT)

*Corresponding author; e-mail: J.Ferkl@rcmt.cvut.cz

\begin{abstract}
Telescopic covers are components which can have a potentially large impact on machine tool feed drive capabilities. Generally speaking, frictional force modelling is a well-established field of interest in which many approaches and challenges have been identified. This paper addresses frictional force modelling of machine tool feed drive telescopic covers. A sequential assembly approach and control system data acquisition are used for friction model parameter estimation and verification. Models of telescopic covers may be used in virtual machine tools. Furthermore, feed drive accuracy and dynamics may be enhanced by compensation of frictional forces in the control system. Other possible applications include component commissioning during machine tool assembly and predictive maintenance.
\end{abstract}

\section{Keywords:}

Machine Tool; Feed Drive; Telescopic Covers; Virtual Machine Tool; Friction Modeling; LuGre Model

\section{INTRODUCTION}

Feed drive motion accuracy and dynamics affect machine tool performance directly. Friction is defined as 'a resistance which opposes the relative motion of contacting bodies' [CIRP 2020]. It simultaneously affects the feed drive positively as a source of damping and negatively as an external load. In the case of rolling elements, resistance to relative movement is typically much lower and steadily increases with velocity. Thus, the moving components in a feed axis closed force loop are typically based on rolling elements - a ball bearing, ball screw, rolling guideways, etc. On the other hand, the primary purpose of a telescopic cover for a machine tool feed drive is not closing the force loop but rather mechanically protecting the aforementioned components from the cutting process - namely, the removed material and cutting fluid. Telescopic cover segments do not have to be excessively rigid, but they do have to seal across the entire axis travel. Telescopic covers with kinematically synchronized segments are characterized by significant friction, which is caused by the many relative movements that are present.

\subsection{Frictional Force Modeling}

By definition, the value of a frictional force depends on the addressed motion. The Stribeck curve models frictional force as a static function of velocity [Stribeck 1901]. Its main drawbacks are discontinuity at zero velocity and neglected hysteretic behaviour. The first issue can be addressed by a very steep increase in the absolute value in the near zero region as shown in [Armstrong 1995]. The first model addressing hysteresis describes frictional force as a function of displacement [Dahl 1968]. Resistance to motion in the near zero velocity region is approached as the deformation of surface asperities in contact, described by a stress-strain diagram. The Dahl model does not include the Stribeck effect, but it laid the foundation for subsequent models addressing the near zero velocity region as a presliding regime where frictional force depends on displacement rather than velocity.

The LuGre model expands on the idea of deflection of surface asperities, which is represented by the state variable $z$ [Canudas 1995]:

$\frac{d z}{d t}=v-\frac{|v|}{g(v)} z$

Where $v$ is relative velocity between moving surfaces in contact. The value of $z$ in the steady state approach value of the function $g(v)$ [Canudas 1995]:

$\sigma_{0} g(v)=F_{C}+\left(F_{S}-F_{C}\right) e^{-\left(\frac{v}{v_{S}}\right)}$

Where $\sigma_{0}$ is asperity stiffness, $F_{C}$ is Coulomb friction, $F_{S}$ is stiction force and $v_{S}$ is Stribeck velocity. The resulting frictional force $F$ is the superposition of asperity stiffness, asperity damping ( $\sigma_{1}$ coefficient) and viscous friction represented by the linear dependency of the frictional force on the relative velocity $v$ ( $\sigma_{2}$ coefficient) [Canudas 1995]:

$F=\sigma_{0} z+\sigma_{1} \frac{d z}{d t}+\sigma_{2} v$

The LuGre model is a system of equations and as such does not feature memory. This fact results in an inability to 
create and close inner hysteresis loops, as pointed out in [Swevers 2000]. Swevers proposes a new model called the 'Leuven model', which contains two memory stacks storing past extremums to allow the formation of inner hysteresis loops. The working principle is described in detail in the referenced publication [Swevers 2000] and later revisited in [Lampaert 2002]. Another advantage of the Leuven model over the LuGre model is the possibility of defining a transition curve as an arbitrary point-symmetrical, strictly increasing function, whereas the transition curve of the LuGre model is given by model structure [Swevers 2000].

Lampaert et al. presented the Leuven model enhanced by a mechanism called the 'Maxwell slip model' [Lampaert 2002]. It models the frictional force transition curve as a set of parallel elements with various stiffness spring connections to a common node. The acting force upon every element corresponds to its stiffness and common displacement. As the displacement and forces increase, more elements go from sticking to sliding, the resulting system stiffness decreases, and a transition curve is formed. Sticking and slipping of elements corresponds to the Coulomb slip law. This mechanism was further advanced by Al-Bender et al. [Al-Bender 2005], where the Coulomb slip law is replaced by a 'weighted Stribeck velocity weakening'. This model is called the 'Generalized Maxwell slip model'.

Rebelein et al. examined individual feed drive components as local damping sources using the Leuven model [Rebelein 2016] followed by [Rebelein 2017] [Zaeh 2019] [Semm 2020]. The accurate results of the assembled feed axis dynamics display potential, but the telescopic cover is not included among the identified components. Two approaches to identify friction properties are presented the sequential assembly process and the test bench approach. Kolar examined the frictional force of a telescopic cover using the integrated Coulomb and viscous friction model with the help of the finite element method (FEM) and experimental measurements [Kolar 2010]. Further model complexity was achieved by modelling the segments of a studied telescopic cover as interacting compliant bodies [Kolar 2011]. Wang presented the LuGre model parameter identification method based on an evolutionary algorithm [Wang 2016]. There are many other publications presenting application and variations of these models [Yoon 2014] [Freidovich 2010] [Villegas 2014].

The LuGre model is used often despite its disadvantages in comparison to the Leuven model as mentioned above. The implementation and low computational requirements of the LuGre model are both great advantages. It is also effective in telescopic cover frictional force modelling, as shown in Section 4 . The key focus of this paper is the transferability of knowledge into industrial practice. Thus, the LuGre model is used as a targeted approach with remarkable accuracy in the examined application.

\subsection{Paper objectives and structure}

Frictional force modelling is a well-established field of interest with many advanced models. The aim of this paper is not to extend existing theories of friction as a physical phenomenon; rather, the goal is to identify the behaviour of the telescopic cover as a component and describe its impact on feed axis behaviour. This knowledge can serve as part of a virtual machine tool [Altintas 2005] [Kolar 2019] as a concept for frictional force compensation in the control system, for the purposes of predictive maintenance and also for quality verification of the examined component during the machine tool assembly and commissioning process. The frictional force identification method used herein requires no additional sensors.

The complete feed axis model is assembled sequentially in three stages. In the final stage, the difference between the measured power required to accomplish the defined movement cycles is ascribed to the frictional force of the telescopic cover. Fig. 1 illustrates the approach in detail.

This procedure can be understood as a nonlinear least squares problem. Generally, defining the nonlinear function to be fitted into the measured data is crucial. In the presented strategy, the feed axis simulation model is the

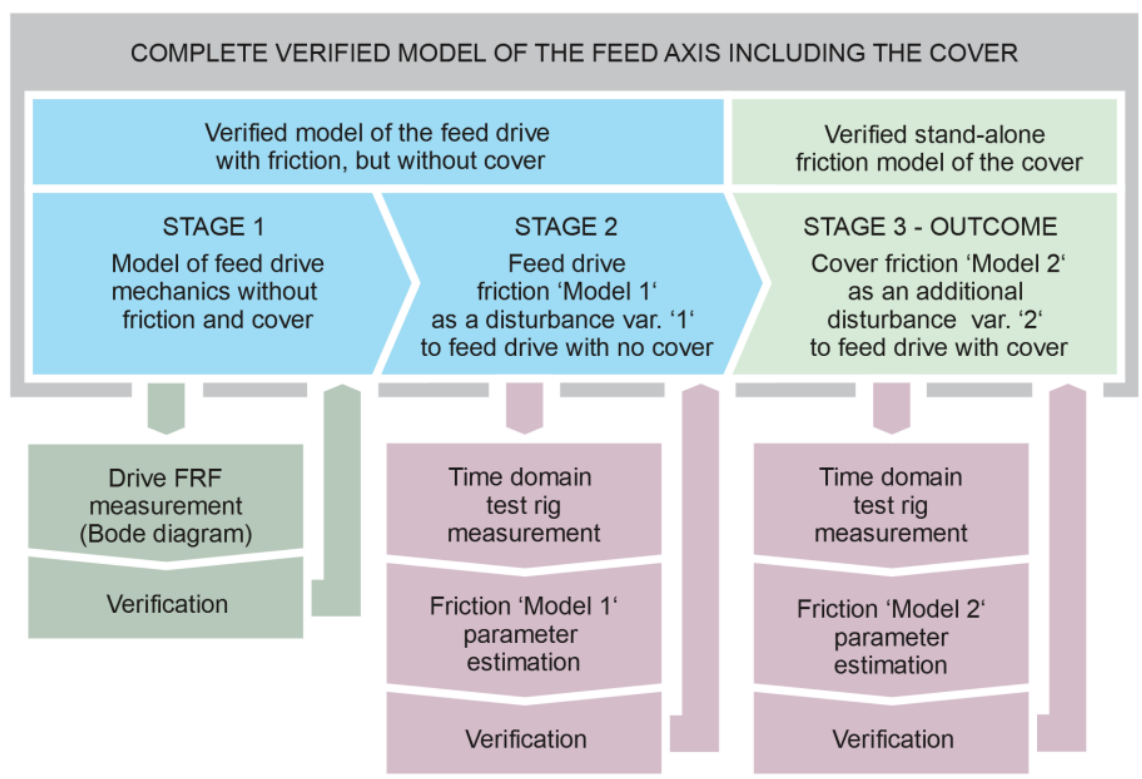

Fig. 1: Strategy for obtaining a verified frictional force simulation model of the feed axis cover 


\section{HSM \\ 2021 \\ MM Science Journal | www.mmscience.eu \\ ISSN 1803-1269 (Print) | ISSN 1805-0476 (Online) \\ Special Issue | HSM 2021 \\ $16^{\text {th }}$ International Conference on High Speed Machining \\ October 26-27, 2021, Darmstadt, Germany}

DOI: 10.17973/MMSJ.2021_11_2021163

nonlinear function. Two separate friction models are included in this simulation model. Thus, the friction parameters are the arguments of this function. These arguments are tuned to minimize the sum of squared residuals between the simulated function values and the measured data using the 'trust-region' algorithm described in Section 4.

Stage 1 is devoted to the creation and verification of a mechanical model and the control system of the feed axis. The model structure is described in detail in Section 3. The initial values of the model parameters are based on the physical properties of the individual components listed in the datasheets and drawings. Control system simulation is appended and the frequency response function (FRF) of the mechanical system is simulated. Component stiffness and damping parameters are estimated and verified based on a comparison with the experimentally measured FRF on the test rig.

Stage 2 involves modelling of the frictional force, which represents the resistance to the movement of the feed axis without a telescopic cover. 'Model 1' of the frictional force (Fig. 1) is added and the initial parameters are roughly estimated. The subsequent algorithmic parameter estimation relies on time domain signal analysis. The experimental test rig is operated under different conditions while data from the control system are acquired. The recorded position setpoint serves as the simulation input and the simulated actual torque is curve fitted to the measured actual torque by tuning the friction Model 1 parameters. At this point, the simulation model of the feed axis without a telescopic cover is complete, but the friction impact on the FRF must be considered. The model of feed axis mechanics with no friction model was verified with the experimental test rig FRF, where friction is present. Consequently, Stages 1 and 2 have to be repeated at least once. In the case of a nonlinear frictional force model (e.g., LuGre), the FRF of the simulation model cannot be carried out as a linearization. Instead, it necessitates time domain simulation.

In Stage 3, a second frictional force model called 'Model 2' (Fig. 1) is appended to the verified model of the feed axis with the drive friction included. The parameter estimation process described above is repeated, but this time, the experimental test rig is operated with the telescopic cover. As a result, the measured actual torque increases. The simulated actual torque is once again curve fitted with tuning of Model 2 parameters.

\section{EXPERIMENT SETUP}

The experimental test rig (see Fig. 2) consists of a ball screw fixed at one end in the bearing and free at the other end (fixed-free shaft mounting method). The axial stiffness of the bearing is $19 \cdot 10^{8}[\mathrm{~N} / \mathrm{m}]$. The ball screw nominal diameter and lead is $32[\mathrm{~mm}]$. The length of the thread is $850[\mathrm{~mm}]$. The axial stiffness of the ball screw nut is 3 . $10^{8}[\mathrm{~N} / \mathrm{m}]$. The ball screw shaft is $112[\mathrm{~mm}]$ long. It is coupled with a servo module by a coupling with a torsional stiffness of $17800[\mathrm{Nm} / \mathrm{rad}]$ and moment of inertia of 114 . $10^{-6}\left[\mathrm{kgm}^{2}\right]$. The moment of inertia of the rotor is 593 . $10^{-6}\left[\mathrm{kgm}^{2}\right]$. The servo module features a $24 \mathrm{bit}$ rotary encoder that is used for both velocity and position control loop feedback. Linear rolling guideways are used for the table and the cover is guided by separate slideways. The positioned table is loaded with an additional rigid mass and a welded console (38 $[\mathrm{kg}])$ to which the telescopic cover is fastened (see Fig. 2). The total weight of the loaded table is $106[\mathrm{~kg}]$ including the aforementioned console. The telescopic cover consists of 6 segments that cover a stroke length of $400[\mathrm{~mm}]$. The cover corresponds to the current industry standard. The movement of the cover segments is kinematically bound by a scissor mechanism.

The feed axis on the test rig was operated in a reversing sequence consisting of two sequential acceleration ramps followed by two sequential deceleration ramps. The velocity stabilizes after the first acceleration and deceleration ramp at half value and also at the maximum value after the second acceleration ramp (Fig. 3). The acceleration of the axis was constant at a value of $10\left[\mathrm{~m} / \mathrm{s}^{2}\right]$. Values of maximum velocity were randomly generated at a specified interval. The intervals are described in Section 4. Naturally, the first acceleration ramp starts at zero velocity and the second acceleration ramp does not. This difference is valuable for frictional force identification because the inertial force in the case of identical acceleration remains the same, but the frictional force changes as it depends on
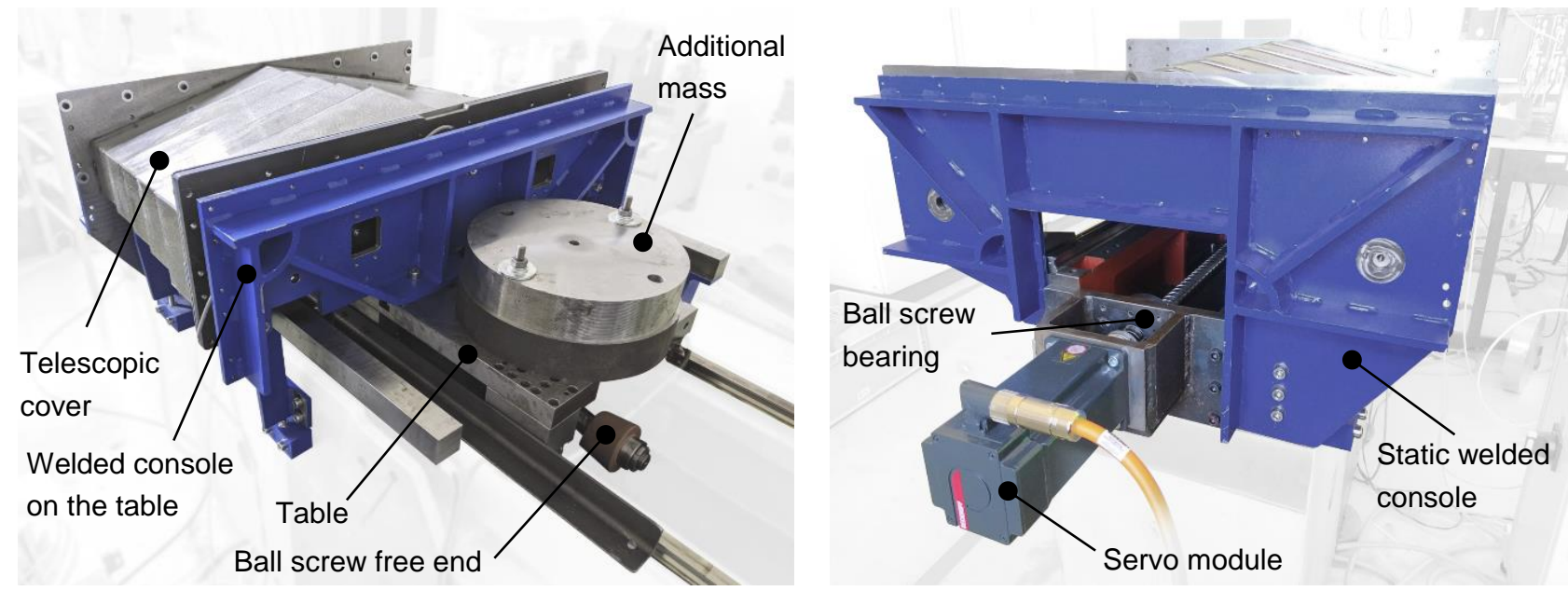

Fig. 2: The experimental test rig 
displacement in the pre-sliding regime and velocity in the sliding regime.
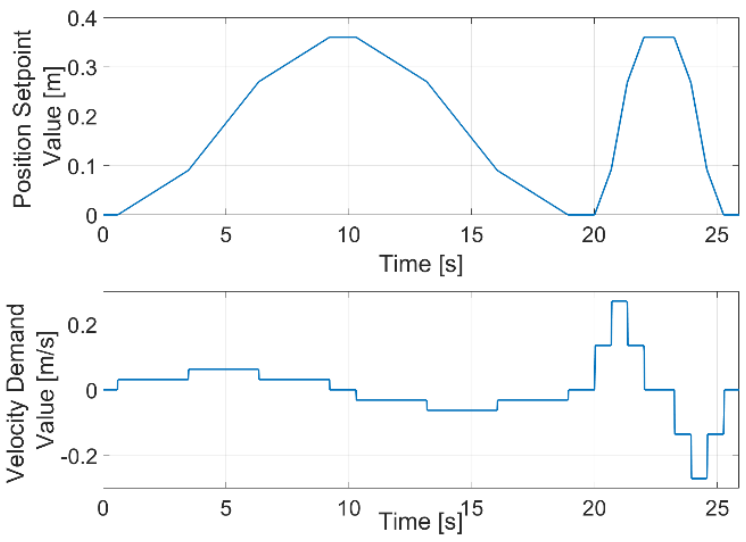

Fig. 3: Two cycles of the reversing sequence

Cascade controller P-position, PI-velocity and PI-current are used with all control loops running at a frequency of $16[\mathrm{kHz}]$. The control system platform is Beckhoff TwinCAT 3 , servo module AX8000. A first order low-pass filter and band-stop filter are present at the current control loop input. All feed forwards are turned off. The complete Beckhoff control system is replicated as an integral part of the simulation model. The setpoint values and actual values of all three control loops during the motion cycles (Fig. 3) on the experimental test rig are acquired at sampling frequency $1[\mathrm{kHz}]$.

\section{SIMULATION MODEL DESCRIPTION}

The mechanics simulation model is implemented in the Matlab - Simscape simulation environment. Mathematical expressions are defined as a part of the source code of individual blocks. The model is assembled as a circuit of connected blocks interacting with each other by means of a through variable (force) and an across variable (velocity). The working principle is analogous to an electrical circuit, where the through variable is the current and the across variable is the voltage. A diagram of the simulation model is shown in Fig. 4.

The ball screw is modelled using two branches formed by FEM beam elements - rotational and translational. The rotational branch represents the rotation of the ball screw and torsional deflection. The translational branch represents the axial deflection. Each branch is composed of eight elements. Four of them stand for the active part of the thread between the motor and the ball screw nut. The remaining four elements represent the passive part that does not participate in the closing of the force loop. The length of the active and passive sections (corresponding FEM elements) is continuously recalculated as the positioned table travels. Use of FEM elements instead of lumped masses enables modelling of a continuous distribution of mass throughout the element through the implementation of consistent mass matrices [Logan 2007]. The damping matrix was derived as the linear combination of mass and stiffness matrices (coefficients $c_{i}$ ):

$m\left[\begin{array}{cc}\frac{1}{3} & \frac{1}{6} \\ \frac{1}{6} & \frac{1}{3}\end{array}\right]\left[\begin{array}{l}\ddot{x}_{1} \\ \ddot{x}_{2}\end{array}\right]+\boldsymbol{B}\left[\begin{array}{l}\dot{x}_{1} \\ \dot{x}_{2}\end{array}\right]+k\left[\begin{array}{cc}1 & -1 \\ -1 & 1\end{array}\right]\left[\begin{array}{l}x_{1} \\ x_{2}\end{array}\right]=\left[\begin{array}{l}F_{1} \\ F_{2}\end{array}\right]$

$\boldsymbol{B}=c_{1} m\left[\begin{array}{cc}\frac{1}{3} & \frac{1}{6} \\ \frac{1}{6} & \frac{1}{3}\end{array}\right]+c_{2} k\left[\begin{array}{cc}1 & -1 \\ -1 & 1\end{array}\right]$

$J\left[\begin{array}{cc}\frac{1}{3} & \frac{1}{6} \\ \frac{1}{6} & \frac{1}{3}\end{array}\right]\left[\begin{array}{l}\ddot{x}_{1} \\ \ddot{x}_{2}\end{array}\right]+\boldsymbol{B}_{T}\left[\begin{array}{l}\dot{x}_{1} \\ \dot{x}_{2}\end{array}\right]+k_{T}\left[\begin{array}{cc}1 & -1 \\ -1 & 1\end{array}\right]\left[\begin{array}{l}x_{1} \\ x_{2}\end{array}\right]=\left[\begin{array}{l}T_{1} \\ T_{2}\end{array}\right]$

$\boldsymbol{B}_{T}=c_{3} J\left[\begin{array}{cc}\frac{1}{3} & \frac{1}{6} \\ \frac{1}{6} & \frac{1}{3}\end{array}\right]+c_{4} k_{T}\left[\begin{array}{cc}1 & -1 \\ -1 & 1\end{array}\right]$

The two branches (rotational and translational) are connected through the transformer. The resulting motion and force interact with the table through the ball screw nut modelled as a spring and a damper. The table itself is modelled as a discrete mass. The console was modelled as two parallel masses on a spring and damper. It is based on a comparison of the FRF of the experimental test rig with and without the welded console. The total mass of the table corresponds to reality. Additional springs and dampers (Fig. 4) were tuned to obtain a simulated FRF similar to the measured FRF. There are two blocks representing friction. Model 1 represents the friction of the whole drive. Model 2 represents the friction of the telescopic cover. There is an optional input for cutting force implementation.

As mentioned above, the ball screw is fixed at one end in the bearing. This fact is translated into the simulation model by linking the active end of the ball screw to the frame through the spring of the axial stiffness of the bearing. The rotational branch is not fixed, because the ball screw has to be free to rotate. The moment of inertia of the rotor and half of the coupling is represented as a lumped inertia $\left(J_{1}\right)$. The ball screw shaft end is represented by the FEM element of constant length. It is directly connected to the lumped

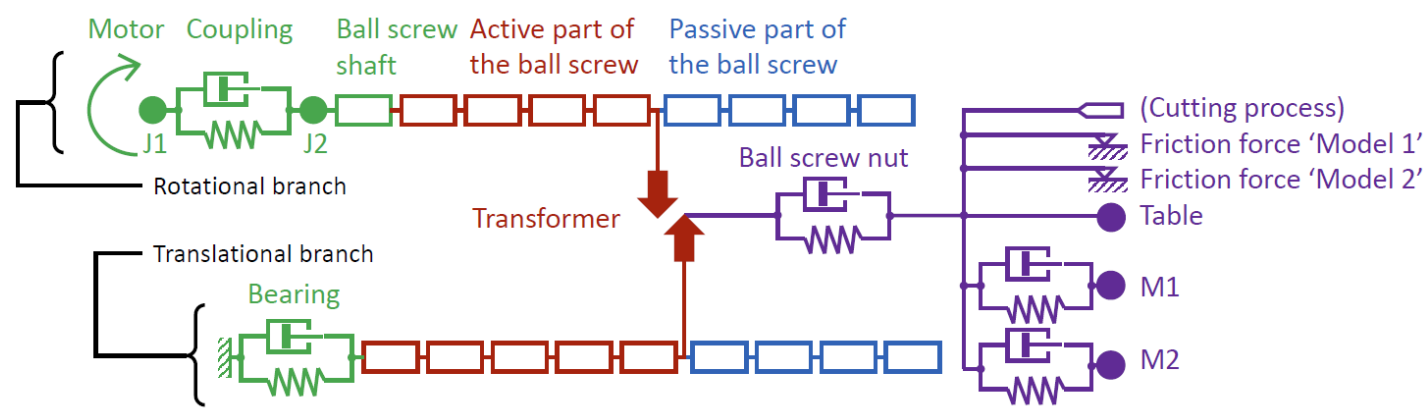

Fig. 4: Block diagram of the linear feed axis simulation model

MM Science Journal | 2021 | NOVEMBER - Special Issue on HSM2021 
moment of inertia, which represents the remaining half of the coupling $\left(J_{2}\right) . J_{1}$ and $J_{2}$ are connected through the spring and damper representing the coupling. The servo module is represented by an ideal source of torque driving the rotational branch and is controlled by the cascade controller. The structure and parameters of the cascade controller in the model correspond to the controller in the AX8000 servo system on the test rig.

\section{FRICTIONAL FORCE MODEL PARAMETER ESTIMATION AND VERIFICATION}

Stage 1 serves for verification of the simulation model of mechanics and controls. The FRF of the closed velocity control loop of the experimental test rig was measured and compared with the closed velocity control loop FRF of the simulation model (see Fig. 5). During the measurement, the experimental test rig was positioned in the middle of the feed axis stroke length. This fact was reflected in the simulation model as an initial condition. The stiffness values from the datasheets and estimated damping values were tuned to achieve a better coincidence.

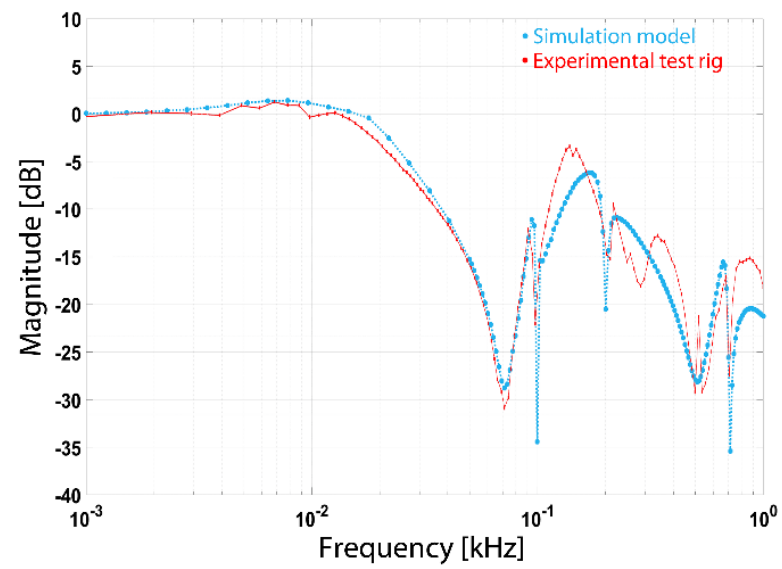

Fig. 5: The FRF of the closed velocity control loop

In Stage 2, the experimental test rig was operated in the reverse motion sequence. The maximum velocity values were generated using a random number generator in three specified intervals. The travel distance was adjusted with respect to the prescribed velocity:

1. 50 cycles of the travel length $0.36[\mathrm{~m}]$ were measured in the maximum velocity interval $<0.05 ; 0.4>[\mathrm{m} / \mathrm{s}]$.

2. 50 cycles of the travel length $0.05[\mathrm{~m}]$ were measured in the maximum velocity interval $<0.005 ; 0.05>[\mathrm{m} / \mathrm{s}]$.

3. 20 cycles of the travel length $0.005[\mathrm{~m}]$ were measured in the maximum velocity interval $<0.0001 ; 0.005>[\mathrm{m} / \mathrm{s}]$.

The simulation model was extended by frictional force Model 1. The recorded interpolated position setpoint was used as the simulation model input. The simulated torque required to accomplish the defined motion was curve fitted to the measured torque using the 'trust-region' algorithm [Branch 1999] [Byrd 1988]. It is an iterative process minimizing a defined cost function $f(\boldsymbol{x})$. In every iteration, function values are approximated by a simple expression. The neighbourhood of the current value of $\boldsymbol{x}$, where the approximation is expected to satisfy the required accuracy, is called the trust-region. The local minimum of the approximation in the trust-region is determined and the value of the cost function is calculated. If the newly found cost function value is smaller than the previous one, the current value of $\boldsymbol{x}$ is updated. Otherwise, the trust-region is reduced. For curve fitting of the measured torque, the cost function is calculated as a sum of the squared residuals between the simulated and measured actual torque. The vector argument $\boldsymbol{x}$ stands for the friction model parameters which are to be determined. This process corresponds to a nonlinear least squares data-fitting problem.

Interval $1 \quad(<0.05 ; 0.4>[\mathrm{m} / \mathrm{s}])$ was used for the estimation. The complete record is more than $9[\mathrm{~min}]$ long, which is not suitable for the iterative process. Fig. 6 a) shows the section that was used for curve fitting. The frictional force was recalculated to the torque using the ball screw lead to achieve comparable results. A curve fitting algorithm converged as the local minimum of the cost function was found (see Tab. 1). Subsequently, the simulation model was verified by a comparison of the measured and simulated torques in the course of the complete measurements of intervals 1, 2, (see example section Fig. 6 b) ) and 3 (see example section Fig. 6 c) ).

We may conclude that the estimation process was successful, as the simulated total torque (Fig. 6 a) red curve) fitted in with the measured total torque (Fig. 6 a) yellow curve). This statement is also true for the data that were used for verification rather than for estimation. There are a few discrepancies. They are discussed in detail in Section 5. Stage 1 and Stage 2 were repeated once to take into consideration friction during the FRF comparison. The figures and parameters presented here correspond to the second iteration.

Next, Stage 3 was carried out. The experimental test rig was operated in reverse sequence measurements with the telescopic cover fastened to the welded console. Motion setpoint values were once again generated by a random number generator. The same maximum velocity intervals and travel length were used as in the previous stage. Frictional force Model 2 was connected to the simulation model. The curve fitting algorithm was once again successful, fitting two cycles from interval $1(<0.05 ; 0.4>$ $[\mathrm{m} / \mathrm{s}]$, see Fig. 7 a) ). The simulation model with the estimated parameters of both frictional force models was verified through a comparison with the complete measurements of all intervals (see Fig. 7 b) and c) ). The estimated parameters are presented in Tab. 1.

Tab. 1: Estimated parameters of both frictional force models

\begin{tabular}{|c|c|c|}
\hline Variable & Model 1 & Model 2 \\
\hline$F_{S}[N]$ & 200 & 49.6 \\
\hline$F_{C}[\mathrm{~N}]$ & 163 & 106 \\
\hline$v_{S}[\mathrm{~m} / \mathrm{s}]$ & $2.53 \cdot 10^{-3}$ & $5.30 \cdot 10^{-4}$ \\
\hline$\sigma_{0}[\mathrm{~N} / \mathrm{m}]$ & $9.37 \cdot 10^{6}$ & $1.92 \cdot 10^{6}$ \\
\hline$\sigma_{1}[\mathrm{Ns} / \mathrm{m}]$ & $4.45 \cdot 10^{4}$ & $5.78 \cdot 10^{3}$ \\
\hline$\sigma_{2}[\mathrm{Ns} / \mathrm{m}]$ & 530 & 86.2 \\
\hline
\end{tabular}



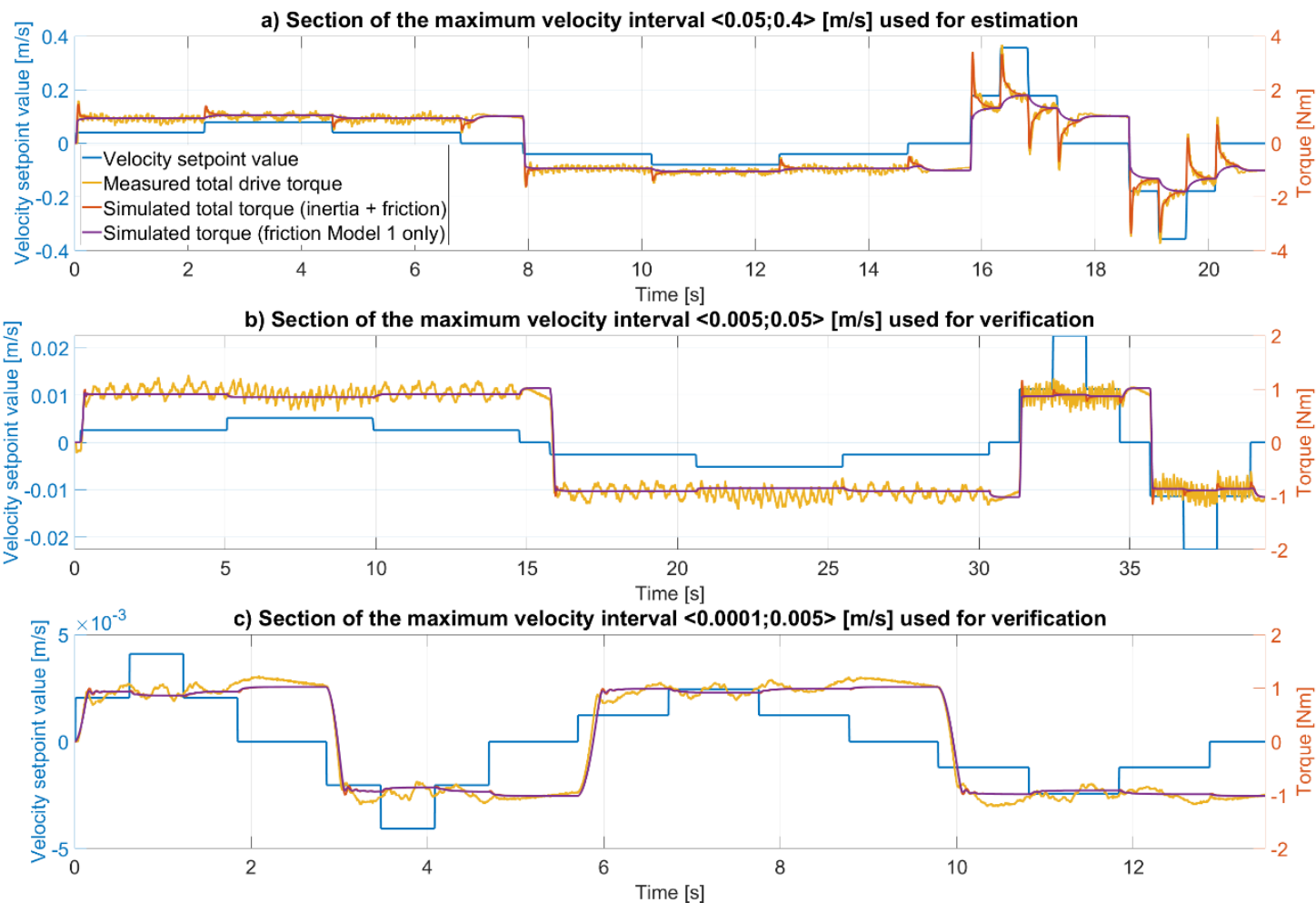

Fig. 6: Stage 2 (movement without cover): a) parameter estimation; b), c) parameter verification
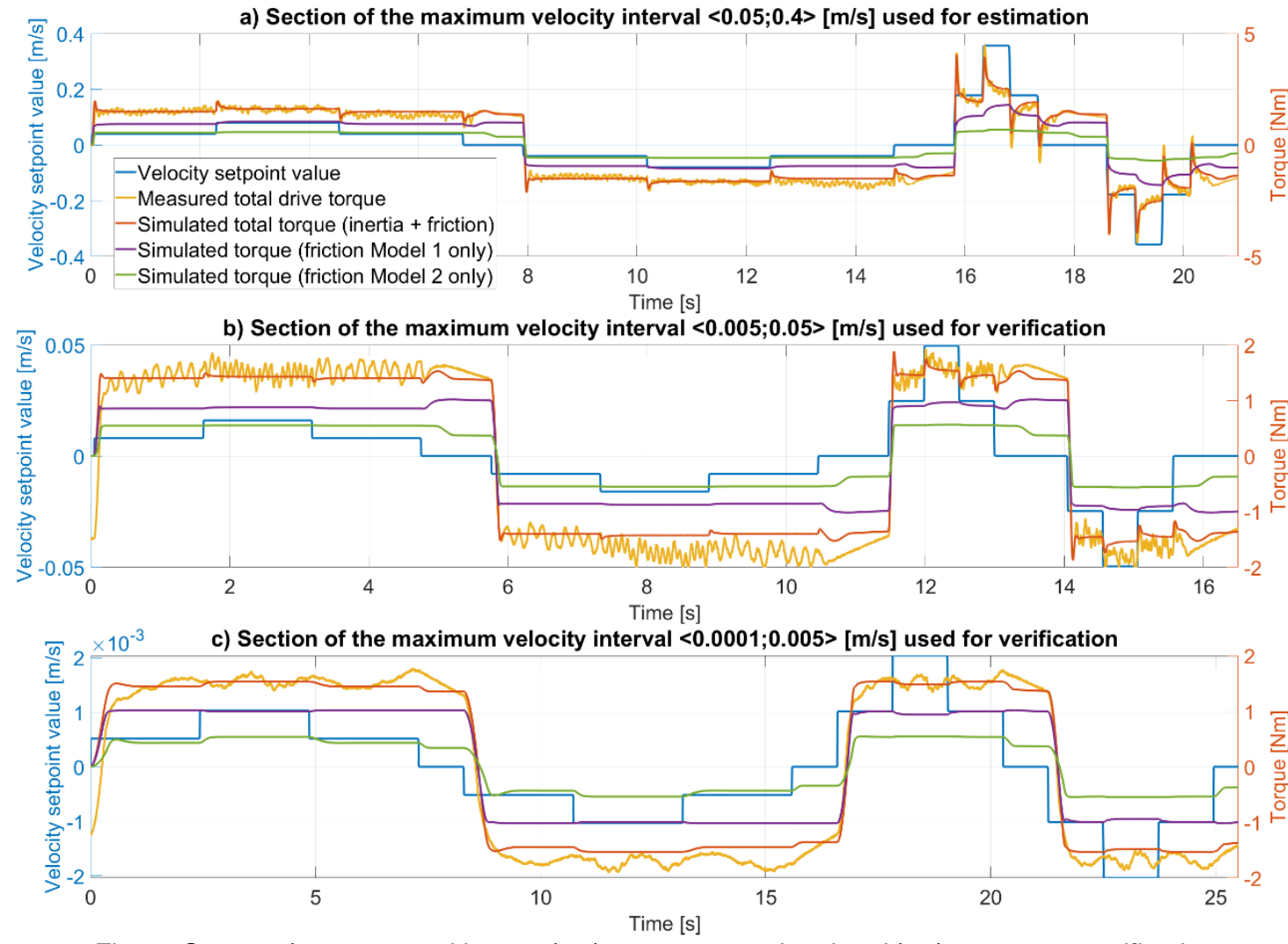

Fig. 7: Stage 3 (movement with cover): a) parameter estimation; b), c) parameter verification 


\section{DISCUSSION}

A simulation model of feed drive mechanics without a telescopic cover but with the friction of the drive was designed and verified. A separate friction model representing the telescopic cover friction was added and the parameters were estimated. The verification proved superb coincidence between the measured and simulated data, even though there are some simplifications in the simulation model. Neither the servomotor poles nor the ball screw rolling elements were considered in the simulation model. Consequently, the resulting simulated torques do not oscillate as the measured actual torque signal. The inertial forces bounded with the telescopic cover are neglected. The overall feed drive friction is represented as a single frictional force acting upon the positioned table. It is not ascribed to the individual components. The frictional force simulation model is symmetrical. Thus, the difference between the opening and closing of the telescopic cover due to wipers orientation is not taken into account. The indirect measurement used as the position control loop feedback is less accurate than the direct measurement.
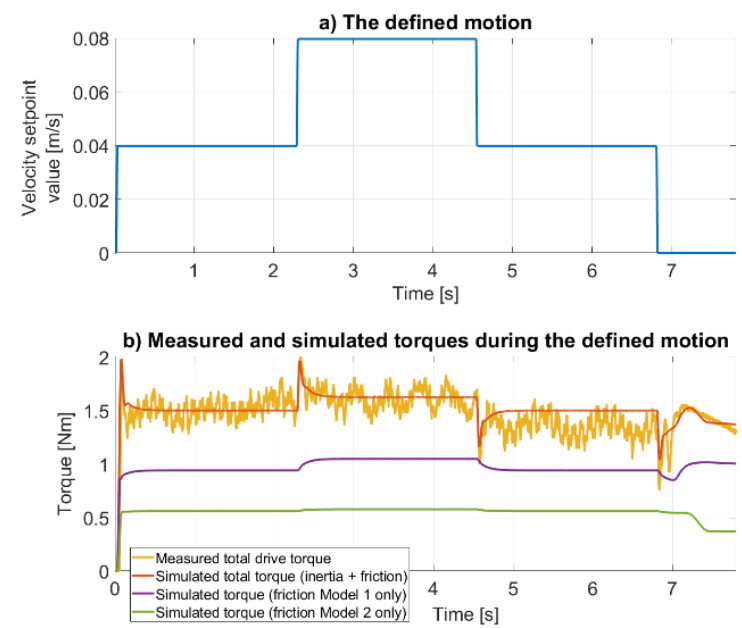

c) Share of the simulated cover friction force (Model 2) on the total torque

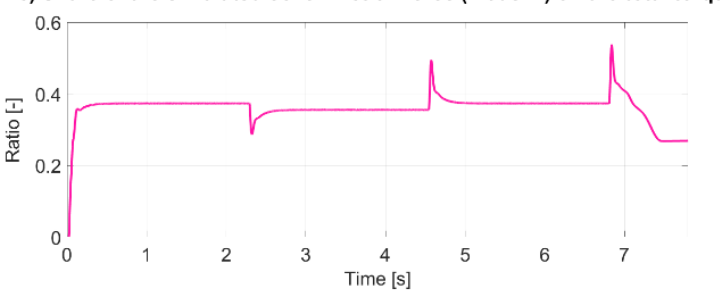

Fig. 8 : Motion in positive direction a) common velocity setpoint for both the experimental test rig and the simulation model; b) measured and simulated torques;

c) share of the telescopic cover frictional force on simulated total torque

There are a few discrepancies between the measured and simulated total torque signals which emerged from the time domain verification in both Stage 2 and Stage 3 . The frictional force asymmetry mentioned above is obvious during the negative velocity value motion, as the frictional force is bigger (see Fig. 7 b) and c) ). This corresponds to the closing of the telescopic cover. Naturally, the resulting approximation accuracy is affected in both motion directions. Another shortcoming of the simulation model is present in the zero velocity region. The frictional force at a standstill depends on previous motion due to the hysteretic nature of friction. This phenomenon is apparent in the actual torque value as the examined feed axis stops. During the reverse sequence used in Stage 2 and Stage 3, there is 1 [s] idle time in every reverse motion (see Fig. 6 and Fig. 7). Fig. 8 presents a detailed view of the motion in the positive direction and following standstill state. It can be seen that the frictional force for the experimental test rig tends to fade out in standstill. The LuGre model is unable to represent this behaviour.

Apart from the simulation model, there is also potential for improvement in the parameter estimation strategy. The LuGre model parameters were estimated in a relatively high velocity region (see Section 4). This can be advantageous for parameters such as $\sigma_{2}$ describing viscous friction, but simultaneously disadvantageous for parameters bounded with near zero velocity behaviour such as $v_{S}$. The resulting inaccuracy is observable in Fig. 6 b) at time 21 [s] as the simulated friction torque decreases and the measured total torque increases as a consequence of a velocity increase.

To interpret the estimated telescopic cover friction model parameters, the resulting cover frictional force is expressed as a fraction of the total torque required to achieve the defined motion (see Fig. $8 \mathrm{c}$ )). In steady state, the studied telescopic cover is responsible for almost $40 \%$ of the total torque requirements. Such a significant share proves the telescopic cover's significant impact on the feed axis drive. The importance of the cover is amplified as there is no cutting process and the positioned table is relatively lightweight.

\section{CONCLUSION}

This paper proposes a strategy for estimation of friction model parameters. It is based on a sequential assembly approach and curve fitting of the machine tool feed drive control system time domain data. In this paper, friction induced by a telescopic cover was identified. The LuGre friction model was used, but the proposed strategy may be generalized to identify the parameters of an arbitrary friction model.

The approach described in this paper provided very accurate experimental results. The significant importance of the investigated telescopic cover component on the test rig was demonstrated.

\section{ACKNOWLEDGEMENT}

The authors would like to acknowledge funding support from the Czech Ministry of Industry and Trade under the project FV30379 "Energy efficient cover for advanced production machines". The work of one of the authors was also supported by the Grant Agency of the Czech Technical University in Prague, grant no. SGS19/165/OHK2/3T/12.

\section{REFERENCES}

[Al-Bender 2005] AL-BENDER, F., V. LAMPAERT and J. SWEVERS. The generalized Maxwell-slip model: a novel model for friction Simulation and compensation. IEEE Transactions on Automatic Control [online]. 2005, 50(11), 1883-1887 [cit. 2021-5-31]. ISSN 0018-9286. 
[Altintas 2005] ALTINTAS, Y., C. BRECHER, M. WECK and S. WITT. Virtual Machine Tool. CIRP Annals [online]. 2005, 54(2), 115-138 [cit. 2021-6-2]. ISSN 00078506.

[Armstrong 1995] B. Armstrong, C.C. de Wit, Friction Modeling and Compensation, The Control Handbook, CRC Press, 1995.

[Branch 1999] BRANCH, Mary Ann, Thomas F. COLEMAN and Yuying LI. A Subspace, Interior, and Conjugate Gradient Method for Large-Scale Bound-Constrained Minimization Problems. SIAM Journal on Scientific Computing [online]. 1999, 21(1), 1-23 [cit. 2021-6-3]. ISSN 1064-8275.

[Byrd 1988] BYRD, Richard H., Robert B. SCHNABEL and Gerald A. SHULTZ. Approximate solution of the trust region problem by minimization over two-dimensional subspaces. Mathematical Programming [online]. 1988, 40-40(1-3), 247 263 [cit. 2021-6-3]. ISSN 0025-5610.

[Canudas 1995] CANUDAS DE WIT, C., H. OLSSON, K.J. ASTROM and P. LISCHINSKY. A new model for control of systems with friction. IEEE Transactions on Automatic Control [online]. 40(3), 419-425 [cit. 2021-5-31], 1995. ISSN 00189286.

[CIRP 2020] Dictionary of Production Engineering II Material Removal Processes Wörterbuch der Fertigungstechnik II - Trennende Verfahren Dizionario di Ingegneria della Produzione II - Processi di asportazione di materiale [online]. Berlin, Heidelberg: Springer Berlin Heidelberg, 2020 [cit. 2021-5-31]. ISBN 978-3-662-533352.

[Dahl 1968] P.R. DAHL, in: A Solid Friction Model, Aerospace Corporation, El Segundo, 1968, p. 158. Technical Report.

[Freidovich 2010] FREIDOVICH, Leonid, Anders ROBERTSSON, Anton SHIRIAEV and Rolf JOHANSSON LuGre-Model-Based Friction Compensation. IEEE Transactions on Control Systems Technology [online]. 2010, 18(1), 194-200 [cit. 2021-5-31]. ISSN 1063-6536.

[Kolar 2010] KOLAR, Petr, Jan HUDEC and Matej SULITKA. Modeling of Passive Forces of Machine Tool Covers. Journal of Advanced Mechanical Design, Systems, and Manufacturing [online]. 2010, 4(5), 892-901 [cit. 2021 6-4]. ISSN 1881-3054.

[Kolar 2011] KOLAR, Petr, Jan MASEK, Jiri SVEDA and Jan HUDEC. Influence of Machine Tool Covers on Feed Drives. In: ASME 2011 International Manufacturing Science and Engineering Conference, Volume 2 [online]. ASMEDC, 2011, 2011-01-01, s. 57-64 [cit. 2021-6-4]. ISBN 978-07918-4431-1.

[Kolar 2019] KOLAR, Petr, Matej SULITKA, Vojtech MATYSKA and Petr FOJTU. OPTIMIZATION OF FIVEAXIS FINISH MILLING USING A VIRTUAL MACHINE TOOL. MM Science Journal [online]. 2019, 2019(05), 35343543 [cit. 2021-6-2]. ISSN 18031269.

[Lampaert 2002] LAMPAERT, V., J. SWEVERS and F. ALBENDER. Modification of the Leuven integrated friction model structure. IEEE Transactions on Automatic Control [online]. 47(4), 683-687 [cit. 2021-5-31], 2002. ISSN 00189286.
[Logan 2007] LOGAN, Daryl L. A first course in the finite element method. 4th ed. United States: Thomson, c2007. ISBN 978-0-534-55298-5.

[Rebelein 2016] REBELEIN, C. and M. F. ZAEH. Friction in feed drives of machine tools: investigation, modelling and validation. Production Engineering [online]. 2016, 10(4-5), 497-507 [cit. 2021-5-31]. ISSN 0944-6524.

[Rebelein 2017] REBELEIN, C., J. VLACIL and M. F. ZAEH. Modeling of the dynamic behaviour of machine tools: influences of damping, friction, control and motion. Production Engineering [online]. 2017, 11(1), 61-74 [cit. 2021-5-31]. ISSN 0944-6524.

[Semm 2020] SEMM, T., D. SPESCHA, N. CERESA, M.F. ZAEH and K. WEGENER. Efficient Dynamic Machine Tool Simulation with Included Damping and Linearized Friction Effects. Procedia CIRP [online]. 2020, 93, 1442-1447 [cit. 2021-5-31]. ISSN 22128271.

[Stribeck 1901] R. Stribeck , Kugellager Für Beliebige Belastungen, Z. Ver. Deutsch. Ing. 46 (1902) 1341-1348.

[Swevers 2000] SWEVERS, J., F. AL-BENDER, C.G. GANSEMAN a T. PROJOGO. An integrated friction model structure with improved pre-sliding behaviour for accurate friction compensation. IEEE Transactions on Automatic Control [online]. 45(4), 675-686 [cit. 2021-5-31], 2000. ISSN 00189286.

[Villegas 2014] VILLEGAS, Fernando J., Rogelio L. HECKER, Miguel E. PEÑA, Diego A. VICENTE and Gustavo M. FLORES. Modeling of a linear motor feed drive including pre-rolling friction and aperiodic cogging and ripple. The International Journal of Advanced Manufacturing Technology [online]. 2014, 73(1-4), 267-277 [cit. 2021-5-31]. ISSN 0268-3768.

[Wang 2016] WANG, Xingjian, Siru LIN and Shaoping WANG. Dynamic Friction Parameter Identification Method with LuGre Model for Direct-Drive Rotary Torque Motor. Mathematical Problems in Engineering [online]. 2016, 2016, 1-8 [cit. 2021-5-31]. ISSN 1024-123X.

[Yoon 2014] YOON, Jun Young and David L. TRUMPER. Friction modelling, identification, and compensation based on friction hysteresis and Dahl resonance. Mechatronics [online]. 2014, 24(6), 734-741 [cit. 2021-5-31]. ISSN 09574158.

[Zaeh 2019] ZAEH, M.F., C. REBELEIN and T. SEMM. Predictive simulation of damping effects in machine tools. CIRP Annals [online]. 2019, 68(1), 393-396 [cit. 2021-5-31]. ISSN 00078506. 